\title{
Blessures associées aux planches gyroscopiques traitées dans les services d'urgence au Canada : plate-forme électronique du Système canadien hospitalier d'information et de recherche en prévention des traumatismes, 2015-2016
}

\author{
M. T. Do, Ph. D. (1,2); S. McFaull, M. Sc. (1); J. Cheesman (1); T. Mersereau, M. Sc. (3); D. P. Rao, Ph. D. (1); \\ J. Crain, M.A. (1); W. Thompson, M. Sc. (1)
}

Diffuser cet article sur Twitter

\section{Introduction}

Le Système canadien hospitalier d'information et de recherche en prévention des traumatismes $(\mathrm{SCHIRPT})^{1}$ est un système de surveillance des blessures et des intoxications qui a été mis en place en 1990 pour améliorer la disponibilité en données récentes de surveillance au Canada. Le SCHIRPT, financé et administré par l'Agence de la santé publique du Canada, est actuellement utilisé dans 11 hôpitaux pédiatriques et 6 hôpitaux généraux à l'échelle du Canada. Ce compte rendu vise à donner un aperçu des blessures associées aux planches gyroscopiques déclarées au moyen de la plate-forme électronique du SCHIRPT (eSCHIRPT) au 7 juillet 2016.

\section{Méthodologie}

Une planche gyroscopique (aussi appelée " gyroskate ", " planche autoéquilibrante " ou " hoverboard») est un véhicule autoéquilibrant à deux roues semblable à une planche à roulettes, sans guidon et alimenté par pile. Sa vitesse peut atteindre $20 \mathrm{~km} /$ heure. Nous avons réalisé une recherche textuelle dans la base de données de l'eSCHIRPT le 7 juillet 2016 afin de recenser les blessures associées aux planches gyroscopiques. Les événements déclarés ont été trouvés en entrant les expressions suivantes dans le champ de description de la blessure : " hover » (planche gyroscopique) et " self » + " balanc » + " scooter " (" planche " + " auto » + " équilibrante »). Afin que les résultats soient exhaustifs, des termes additionnels ont également été pris en compte (p. ex. " swagway ", " segway ", " planche de hover » et " hover planche »). Nous avons procédé à des vérifications manuelles afin de garantir l'exactitude et la précision des événements trouvés.

\section{Résultats}

La première blessure associée à une planche gyroscopique a été signalée dans l'eSCHIRPT en octobre 2015. En date du 7 juillet 2016, 35 cas avaient été enregistrés dans l'eSCHIRPT (tableau 1). Ces cas étaient répartis également entre hommes et femmes. L'âge moyen au moment de la blessure était de 12,7 ans (ET $=5,0$ ). La plupart des blessures $(n=20)$ ont entrainé une fracture au niveau du bras, du coude, de l'avant-bras ou du poignet (tableau 2). Sur les 35 cas déclarés, 19 ont nécessité des traitements à l'urgence et un suivi. La majorité des blessures se sont produites à l'intérieur $(n=23)$, au cours des mois de décembre et de janvier.

\section{Analyse}

Les planches gyroscopiques, un produit grand public, sont commercialisées au Canada depuis juin 2015. Notre recherche révèle que les planches gyroscopiques ont été utilisées en majorité près de la période des Fêtes. Bien que nos données ne fassent pas mention d'incendies ou d'explosions attribuables aux piles des planches gyroscopiques comme cela est arrivé aux États-Unis ${ }^{2}$, il est surprenant de constater que les planches gyroscopiques ont souvent
TABLEAU 1

Caractéristiques des blessures associées aux planches gyroscopiques

\begin{tabular}{|c|c|}
\hline Caractéristiques & Nombre \\
\hline \multicolumn{2}{|l|}{ Sexe } \\
\hline Masculin & 18 \\
\hline Féminin & 17 \\
\hline \multicolumn{2}{|l|}{ Âge (ans) } \\
\hline 5 à 9 & 6 \\
\hline 10 à 14 & 26 \\
\hline 15 à 19 & 2 \\
\hline 30 à 39 & 1 \\
\hline Moyenne (ET) & $12,7(5,0)$ \\
\hline \multicolumn{2}{|l|}{ Lieu de la blessure } \\
\hline Intérieur & 23 \\
\hline Extérieur & 10 \\
\hline Non indiqué & 2 \\
\hline \multicolumn{2}{|l|}{ Emplacement } \\
\hline Chambre à coucher & 2 \\
\hline Hall, foyer & 3 \\
\hline Cuisine & 2 \\
\hline Salle à manger, cafétéria & 1 \\
\hline $\begin{array}{l}\text { Salon, salle familiale, } \\
\text { salle de jeu }\end{array}$ & 5 \\
\hline Sous-sol, cave & 7 \\
\hline Chaussée, espace pavé & 2 \\
\hline Entrée de cour & 1 \\
\hline $\begin{array}{l}\text { Trottoir, sentier, arrêt } \\
\text { d'autobus }\end{array}$ & 2 \\
\hline Jardin, cour & 4 \\
\hline Emplacement inconnu & 6 \\
\hline
\end{tabular}

Abréviation : ET, écart-type. 
TABLEAU 2

\section{Caractéristiques des blessures}

\begin{tabular}{|c|c|}
\hline Caractéristiques & Nombre \\
\hline \multicolumn{2}{|l|}{ Nature de la blessure } \\
\hline $\begin{array}{l}\text { Blessure superficielle (y compris les } \\
\text { contusions et les abrasions) }\end{array}$ & 2 \\
\hline $\begin{array}{l}\text { Plaie ouverte (y compris les coupures } \\
\text { mineures et les lacérations) }\end{array}$ & 1 \\
\hline Fracture & 20 \\
\hline $\begin{array}{l}\text { Dislocation (y compris les subluxa- } \\
\text { tions vertébrales) }\end{array}$ & 2 \\
\hline $\begin{array}{l}\text { Entorse ou foulure (y compris du } \\
\text { haut et du bas du dos) }\end{array}$ & 2 \\
\hline $\begin{array}{l}\text { Lésion des tissus mous, sans autre } \\
\text { précision }\end{array}$ & 5 \\
\hline Traumatisme crânien fermé mineur & 1 \\
\hline Commotion cérébrale & 2 \\
\hline \multicolumn{2}{|l|}{ Partie du corps } \\
\hline Tête & 3 \\
\hline Bas du dos & 1 \\
\hline Épaule (y compris la scapula) & 1 \\
\hline Bras (y compris l’humérus) & 1 \\
\hline Coude & 4 \\
\hline $\begin{array}{l}\text { Avant-bras (y compris, le radius et } \\
\text { l'ulna) }\end{array}$ & 9 \\
\hline Poignet (y compris le carpe) & 5 \\
\hline Main (y compris les os métacarpiens) & 1 \\
\hline Doigt ou pouce & 4 \\
\hline Genou (y compris la patella) & 2 \\
\hline Cheville (y compris les os du tarse) & 1 \\
\hline Orteils & 3 \\
\hline \multicolumn{2}{|l|}{ Prise en charge } \\
\hline $\begin{array}{l}\text { Conseils seulement, tests diagnos- } \\
\text { tiques, aiguillage vers un omniprati- } \\
\text { cien (aucun traitement à l'urgence) }\end{array}$ & 7 \\
\hline $\begin{array}{l}\text { Traitement à l'urgence avec suivi au } \\
\text { besoin }\end{array}$ & 6 \\
\hline $\begin{array}{l}\text { Observation à l'urgéence avec suivi au } \\
\text { besoin }\end{array}$ & 1 \\
\hline $\begin{array}{l}\text { Observation à l'urgéence, suivi } \\
\text { nécessaire }\end{array}$ & 2 \\
\hline $\begin{array}{l}\text { Traitement à l'urgence, suivi } \\
\text { nécessaire }\end{array}$ & 19 \\
\hline
\end{tabular}

été utilisées à l'intérieur. Leur utilisation dans des lieux confinés devrait être évitée, car elles peuvent causer un incendie en cas de défaillance. En outre, le port d'équipement de protection adéquat est recommandé pendant l'utilisation, notamment un casque, des protège-genoux, des protège-coudes et des protège-poignets. Ces précautions réduiront le risque de fractures, d'entorses ou d'autres blessures en cas de chute.

\section{Limites}

Il est important de signaler que notre échantillon n'est pas pleinement représentatif de la population canadienne. Les adolescents plus âgés et les adultes, les Autochtones et les personnes vivant en milieu rural sont sous-représentés dans la base de données de l'eSCHIRPT car ce système est principalement utilisé par des hôpitaux pédiatriques situés dans de grands centres urbains. Les blessures mortelles sont également sous-représentées dans la base de données de l'eSCHIRPT car les données des services d'urgence n'incluent pas les personnes décédées avant leur transport à l'hôpital ou après leur admission.

\section{Références}

1. Crain J, McFaull S, Thompson W et collab. Rapport d'étape - Le Système canadien hospitalier d'information et de recherche en prévention des traumatismes : un système de surveillance des blessures dynamique et novateur. Promotion de la santé et prévention des maladies chroniques au Canada. 2016;36(6):112-117.

2. US Consumer Product Safety Commission (CPSC). Self-balancing scooters/hoverboards recalled by 10 firms due to fire hazard [Internet]. Bethesda (MD): CPSC; 2016 [consultation le 12 juillet 2016]. Consultable en ligne à la page : http://www.cpsc .gov/en/Recalls/2016/Self-Balancing -Scooters-Hoverboards-Recalled-by-10 -Firms/ 